\title{
Pure nuclear quadrupole resonance determination of the electric field gradient asymmetry for broad lines (and application to $\mathrm{YBa}_{2} \mathrm{Cu}_{3} \mathrm{O}_{7}$ ).
}

\author{
Shahar Levy and Amit Keren \\ Department of Physics, Technion-Israel Institute of Technology, Haifa 32000, Israel.
}

(Dated: October 24, 2018)

\begin{abstract}
We present an angle dependent nuclear quadrupole resonance (ADNQR) method to determine the electric field gradient asymmetry parameter $\eta$ in systems where the resonance line is so broad that the radio frequency field can excite only a portion of the nuclear spins. In this situation the recently developed spectroscopic methods are not applicable. ADNQR is useful for single crystals and oriented powders, and, for small $\eta$ determines $\eta^{4}$. Therefore, it can be used to evaluate fluctuations in $\eta$ due to inhomogeneities. We demonstrate the application of ADNQR experimentally to oriented superconducting $\mathrm{YBa}_{2} \mathrm{Cu}_{3} \mathrm{O}_{7}$ powder.
\end{abstract}

\section{INTRODUCTION}

Pure nuclear quadrupole resonance (NQR) is a very useful tool for studying electronic properties of materials without the need to apply an external magnetic field. One famous example is in the study of superconductors where magnetic fields penetrate the sample in a non uniform way or do not penetrate at all. The NQR is determined by two parameters: a frequency scale $\nu_{q}$ and asymmetry parameter $\eta$ each carry important information on the charge distribution in the system under investigation. However, for nuclei with $I=3 / 2$ there is only one resonance frequency out of which one cannot separate $\nu_{q}$ and $\eta$. In recent years some ingenious experimental methods have been invented to extract these parameters without applying a field. This include 1D [1], and amplitude [2] and phase modulated [3] 2D nutation spectroscopy. However, these methods are based on the assumptions that the NQR line is much narrower than the effective nutation frequency (which is on the order of $\gamma H_{1}$ where $H_{1}$ is the radio frequency (RF) field and $\gamma$ is the nuclear gyromagnetic ratio). Naturally, there are many occasions where these requirements are not met; superconductors, magnetic materials, and systems with disorder are just a few examples.

In this paper we provide an alternative method, based purely on NQR, of determining $\eta$ (and therefore $\nu_{q}$ ) separately for a spin $3 / 2$ in systems with broad lines. In addition, in cases with tetragonal symmetry $(\eta=0)$, our new method is sensitive to the fluctuations in $\eta$, thus providing a measure of the system's homogeneity. However, this method is applicable only to single crystals or oriented powders where all observed nuclei have the same principal axis of the EFG. The main idea of the new method is to measure the signal intensity as a function of the angle between the RF field $\mathbf{H}_{1}(t)$ and the principal axes of the EFG, hence it is named angle depended NQR (ADNQR). In the experiment the sample is rotated with respect to the symmetry axis of the coil.

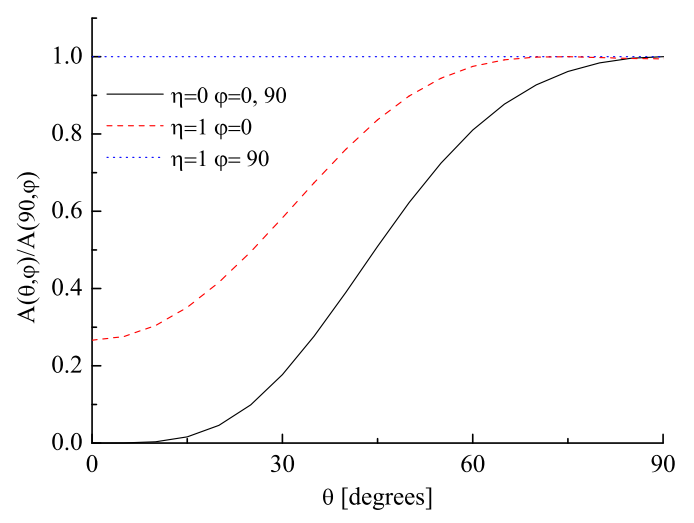

FIG. 1: The expected echo intensity in a single crystal as a function of the polar angle $\theta$ for a fixed azimuthal angle $\varphi$. Two extreme values of $\eta$ and $\varphi$ are examined. The pulse length is optimized at $\theta=90$ and kept constant throughout the sample rotation.

\section{THEORY}

A full theory of the signal intensity as a function of RF field direction was given by Pratt 4]. However, his focus was mainly on the line shape moments in the case of dipolar coupling, and not on the extraction of $\eta$. We therefore re-derive the same calculation from our viewpoint. The starting point is the quadrupole Hamiltonian given by

$$
\mathcal{H}_{q}=\frac{\hbar \nu_{q}}{6}\left[3 I_{z}^{2}-I^{2}+\eta\left(I_{x}^{2}-I_{y}^{2}\right)\right]
$$

As mentioned before, its energy levels are two doublets with angular frequency separation

$$
\omega=2 \pi \nu_{q} \sqrt{1+\frac{\eta^{2}}{3}} .
$$

We further assume that there are no interactions among the nuclear spins and that the line width is due to local 
variations in the values of $\nu_{q}$ and $\eta$, namely, inhomogeneous broadening. In general, such broadening could stamp from crystal defects, impurities, or grain boundaries. In the cuprate superconductors (which will be examined here) the broadening might be due to charge segregation [5]. In these cases of broad lines, a potential use of ADNQR is to scan $\omega$ point by point, determine $\eta$ at each point by ADNQR, and extract $\nu_{q}$ at each point from Eq. 2

The RF field is transmitted in the $\widehat{\mathbf{r}}$ direction on resonance only with a portion of the entire line. The expectation values of the excited spins at time $t$ after the $\mathrm{RF}$ application is obtained by solving

$$
i \hbar \frac{\partial}{\partial t}|n\rangle=\left[\mathcal{H}_{q}-\hbar \omega_{1} \mathbf{I} \cdot \widehat{\mathbf{r}} \cos (\omega t)\right]|n\rangle,
$$

where $\omega_{1}=\gamma H_{1}$. This is done using the matrix

$$
A=\left[\begin{array}{llll}
1 & 0 & -\sigma & 0 \\
0 & 1 & 0 & \sigma \\
\sigma & 0 & 1 & 0 \\
0 & -\sigma & 0 & 1
\end{array}\right]
$$

where $\sigma=\eta /\left[\sqrt{3}\left(1+\sqrt{1+\eta^{2} / 3}\right)\right]$ that diagonalizes $\mathcal{H}_{q}$ and gives

$$
\mathcal{H}_{q}^{A}=\frac{1}{2} \hbar \omega\left[\begin{array}{llll}
1 & 0 & 0 & 0 \\
0 & -1 & 0 & 0 \\
0 & 0 & -1 & 0 \\
0 & 0 & 0 & 1
\end{array}\right]
$$

where the superscript $A$ on any operator $O$ means $O^{A}=$ $A^{\dagger} O A$. By introducing a transformation equivalent to moving into the rotating reference frame in NMR

$$
|n\rangle=A e^{-i \mathcal{H}_{q}^{A} t / \hbar}\left|n^{\prime}\right\rangle,
$$

and substituting Eq. $[5$ in Eq. 3 one finds

$$
i \frac{\partial}{\partial t}\left|n^{\prime}\right\rangle=-\omega_{1} \mathbf{I}^{A}(t) \cdot \widehat{\mathbf{r}} \cos (\omega t)\left|n^{\prime}\right\rangle
$$

where

$$
\mathbf{I}^{A}(t)=e^{i \mathcal{H}_{q}^{A} t / \hbar} \mathbf{I}^{A} e^{-i \mathcal{H}_{q}^{A} t / \hbar} .
$$

Keeping only the secular (time independent) terms of $\mathbf{I}^{A}(t)$ and calling them $\overline{\mathbf{I}^{A}}$, an operation equivalent to ignoring the terms contra-rotating at a frequency $2 \omega$ in NMR, leads to the simplified equation

$$
i \frac{\partial}{\partial t}\left|n^{\prime}\right\rangle=-\omega_{1} \overline{\mathbf{I}^{A}} \cdot \widehat{\mathbf{r}}\left|n^{\prime}\right\rangle
$$

and its solution

$$
\left|n^{\prime}\right\rangle_{t}=\exp \left(i \omega_{1} \overline{\mathbf{I}^{A}} \cdot \widehat{\mathbf{r}} t\right)\left|n^{\prime}\right\rangle_{0}
$$

The RF field is applied for a time period $t_{\pi / 2}$, after which the spins evolve according to $\mathcal{H}_{q}$ only. Therefore

$|n\rangle_{t}=A A^{\dagger} e^{-i \mathcal{H}_{q}\left(t-t_{\pi / 2}\right) / \hbar} A e^{-i \mathcal{H}_{q}^{A} t_{\pi / 2} / \hbar} \exp \left(i \omega_{1} \overline{\mathbf{I}^{A}} \cdot \widehat{\mathbf{r}} t_{\pi / 2}\right)\left|n^{\prime}\right\rangle_{0}$ namely,

$$
|n\rangle_{t}=A e^{-i \mathcal{H}_{q}^{A} t / \hbar} \exp \left(i \omega_{1} \overline{\mathbf{I}^{A}} \cdot \widehat{\mathbf{r}} t_{\pi / 2}\right)\left|n^{\prime}\right\rangle_{0} .
$$

where $t$ is the time measured from the moment the pulse started. In a single coil FID experiment the signal at time $t$ is given by

$$
\begin{aligned}
\langle\mathbf{I} \cdot \widehat{\mathbf{r}}\rangle_{t}^{F I D} & =\frac{1}{Z} \operatorname{Tr} \exp \left(-\beta \mathcal{H}_{q}^{A}\right) \exp \left(-i \omega_{1} \overline{\mathbf{I}^{A}} \cdot \widehat{\mathbf{r}} t_{\pi / 2}\right) \\
& \times\left[\mathbf{I}^{A}(t) \cdot \widehat{\mathbf{r}}\right] \exp \left(i \omega_{1} \overline{\mathbf{I}^{A}} \cdot \widehat{\mathbf{r}} t_{\pi / 2}\right)
\end{aligned}
$$

where $Z$ is the partition function, $\beta=1 / k_{B} T$, and $T$ is the temperature. However, here again we are interested only in terms oscillating at frequency $\omega$. This means that we can replace $\mathbf{I}^{A}(t)$ by

$$
\mathbf{I}^{A}(t) \rightarrow \overline{\mathbf{I}^{A}}(t)=e^{i \mathcal{H}_{q}^{A} t / \hbar} \overline{\mathbf{I}^{A}} e^{-i \mathcal{H}_{q}^{A} t / \hbar} .
$$

$\overline{\mathbf{I}^{A}}(t)$ contains only the oscillating terms at frequency $\omega$ in $\mathbf{I}^{A}(t)$.

For a general irradiation direction, Eq. [6] gives in the high $T$ approximation

$$
\langle\mathbf{I} \cdot \widehat{\mathbf{r}}\rangle_{t}^{F I D}=\frac{\beta \lambda \omega}{2} \sin \left(\lambda \omega_{1} t_{\pi / 2}\right) \sin (\omega t)
$$

where $\lambda$ is an efficiency factor given by

$$
\lambda=\sqrt{r_{x}^{2} a_{x}^{2}+r_{y}^{2} a_{y}^{2}+r_{z}^{2} a_{z}^{2}},
$$

with

$$
\mathbf{a}=\frac{1}{2 \sqrt{3+\eta^{2}}}(\eta+3, \eta-3,2 \eta)
$$

For an echo experiment, obtained by a $\pi / 2-\tau-\pi$ pulse sequence, the phase accumulated during the $\pi / 2$ affects the echo intensity as the 3 rd power of its sinus, as demonstrated for completion in appendix . This leads to our major finding

$$
\langle\mathbf{I} \cdot \widehat{\mathbf{r}}\rangle_{2 \tau}^{E c h o}=\frac{\beta \omega \lambda}{2} \sin ^{3}\left(\lambda \omega_{1} t_{\pi / 2}\right) .
$$

From this point on we will be interested only in the echo amplitude $A$ at time $2 \tau$ as a function of the polar and azimutal angles $\theta$ and $\varphi$ where $\widehat{\mathbf{r}}=$ $(\sin \theta \cos \varphi, \sin \theta \sin \varphi, \cos \theta)$. We therefore define

$$
A(\theta, \varphi)=\langle\mathbf{I} \cdot \widehat{\mathbf{r}}\rangle_{2 \tau}^{E c h o} .
$$

There are two particularly interesting cases: $\varphi=0(x z$ plane) and $\varphi=90$ ( $y z$ plane), for which

$$
\lambda(\theta, \varphi=0)=\sqrt{\frac{(\eta+3)^{2} \sin ^{2} \theta+4 \eta^{2} \cos ^{2} \theta}{4\left(3+\eta^{2}\right)}}
$$


and

$$
\lambda(\theta, \varphi=90)=\sqrt{\frac{(\eta-3)^{2} \sin ^{2} \theta+4 \eta^{2} \cos ^{2} \theta}{4\left(3+\eta^{2}\right)}} .
$$

The echo intensity as a function of $\theta$, for $\varphi=0$ and $\varphi=90$ and for $\eta=0$ and 1 , normalized by the intensity when $\theta=90$, is depicted in Fig. 1 The $\pi / 2$ pulse length is optimized at $\theta=90$ (by $\lambda \omega_{1} t_{\pi / 2}=\pi / 2$ ) and then kept constant throughout the sample rotation. For $\eta \ll 1$ the echo disappears when the RF is applied in the $\widehat{z}$ direction $(\theta=0)$. In addition, there is no difference between the $\theta$ dependence of the echo in the two planes, $\varphi=0$ and $\varphi=90$. On the other hand for $\eta=1$ there is different behavior in the two planes. Along the $y z$ plane $(\varphi=90)$ the echo intensity is constant. In contrast, in the $x z$ plane $(\varphi=0)$ it drops to $27.6 \%$ of its maximum strength.

An important limit which could be further analyzed is $\eta \ll 1$. Since there is no difference between the two planes we denote the efficiency factor at $\theta=90$ by $\lambda_{\perp}=\frac{\sqrt{3}}{2}$. This result was first obtained by Das and Hahn [6]. In this case the optimum pulse length is given by $t_{\pi / 2}=\pi / \omega_{1} \sqrt{3}$ at which the signal amplitude is $A(90, \varphi)=\beta \omega \sqrt{3} / 4$. When keeping the pulse length constant and transmitting in any other direction the relative signal intensity will be

$$
\frac{A(\theta, \varphi)}{A(90, \varphi)}=\frac{2 \lambda}{\sqrt{3}} \sin ^{3}\left(\frac{\pi \lambda}{\sqrt{3}}\right) .
$$

In particular, when transmitting along the $\widehat{z}$ direction $\lambda=a_{z} \simeq|\eta| / \sqrt{3}$, and

$$
\frac{A(0, \varphi)}{A(90, \varphi)}=\frac{2 \pi^{3}}{81} \eta^{4}+O\left(\eta^{6}\right)
$$

This result is important for cases where $\eta$ fluctuates due to crystal inhomogeneity. To appreciate such fluctuations one can determine the averaged $\eta$ by standard methods and the fourth moment of the $\eta$ distribution by ADNQR. Thus measurements of $A(0, \varphi)$ could provide information on the quality of the crystal.

Next we discuss the situation of oriented powders where the $\widehat{z}$ direction is well defined but the $x y$ directions are interchangeable. One example is high temperature superconductors. The samples are usually made from grains of single crystal oriented in a magnetic field so that their $\widehat{z}$ direction aligns with the field direction. However, the $\widehat{x}$ and $\widehat{y}$ directions are random. In this case the outcome of our experiment is a planar average of the result presented in Fig. 1 namely,

$$
A(\theta)=\frac{1}{2 \pi} \int_{0}^{2 \pi} A(\theta, \varphi) d \varphi
$$

This averaging can only be done numerically. In Fig. 2 we present the angular dependence of the echo intensity in

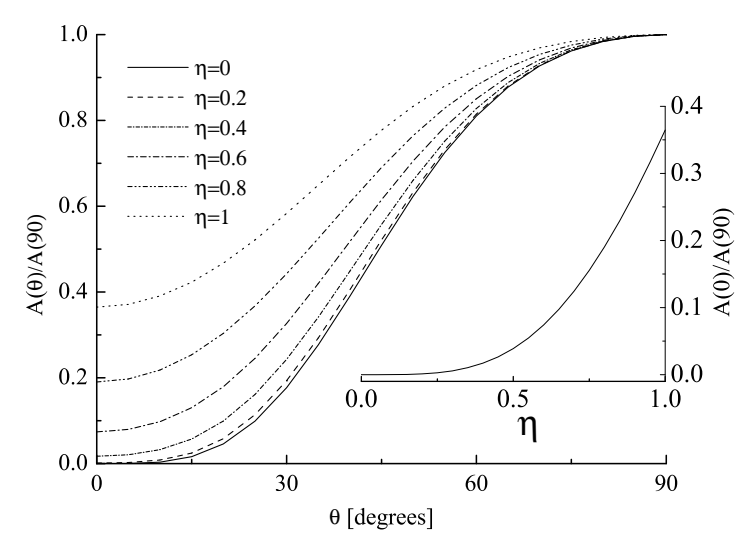

FIG. 2: The expected echo intensity in an oriented powder as a function of the polar angle $\theta$ for various values of $\eta$. The azimuthal angle $\varphi$ is averaged. The pulse length is optimized at $\theta=90$ and kept constant throughout the coil rotation. The inset shows the ratio of the echo intensity between $\theta=0$ and $\theta=90$ as a function of $\eta$.

the case of oriented powder. Here again the pulse length is chosen so that $A(90)$ is optimized. In the $\eta=1$ case, $37 \%$ of the signal remains when $H_{1}$ is in the $\widehat{z}$ direction compared to the signal when $H_{1}$ is in the $x y$ plane. In contrast, in the $\eta=0$ case the signal is lost completely under the same experimental conditions. In the inset of Fig. 2 we show $A(0) / A(90)$ as a function of $\eta$. It is clear that $A(0)$ is most sensitive to $\eta>0.5$.

\section{EXPERIMENT}

We demonstrate an experimental application of this method to ${ }^{63} \mathrm{Cu}$ in oriented powders of the high temperature superconductor $\mathrm{YBa}_{2} \mathrm{Cu}_{3} \mathrm{O}_{7}$ at room temperature. This system has an orthorhombic symmetry, yet all the reports using NMR are consistent with $\eta=0$ [8]. The powder samples are mixed in a teflon container with stycast 1260 and placed in a magnetic field of 9T at room temperature for $24 \mathrm{hr}$. During the first $1 / 2 \mathrm{hr}$ it is shaken with a motor. After drying, a solid cylinder $1.0 \mathrm{~cm}$ in diameter and a $2.0 \mathrm{~cm}$ long sample is removed from the teflon container. In order to obtain the NQR line, the sample is placed in a coil which is tightly wound around it. The NQR line is measured with a Tecmag Apollo spectrometer to which a home-built automated frequency sweep feature is added; the circuit remains tuned and matched throughout the frequency sweep. We find that the NQR line is centered at $31.6 \mathrm{MHz}$ and has a $1 \mathrm{MHz}$ width. These properties and the line shape are in agreement with those previously published [7]. In addition, $T_{2}$ is on the order of $50 \mu \mathrm{sec}$ and depends on the frequency. For the rotation measurements the sample was placed 


\section{ECHO INTENSITY FOR NON PERFECT PULSES}

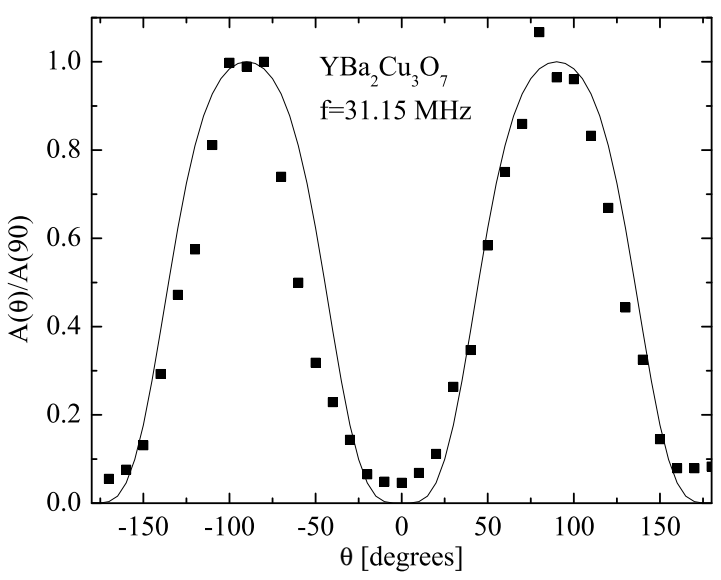

FIG. 3: Experimental demonstration of the variation in echo intensity as a function of $\theta$ in oriented powders of the $\mathrm{YBa}_{2} \mathrm{Cu}_{3} \mathrm{O}_{7}$ superconductor. The solid line is the theoretical expectation for $\eta=0$.

across a $5 \mathrm{~cm}$ long coil to achieve better field homogeneity, and was rotated by hand. In these measurments only $5 \%$ of the line at its center is excited.

We present the rotation data in Fig. 3. We varied $\theta$ over 360 degrees to demonstrate the fact that $A(\theta) / A(90)$ is always positive and periodic. The signal intensity varies by a factor 20 between the $\theta=0$ and $\theta=90$ directions. However, our numerical calculations of Fig. 2 (solid lines) do not fit the experimental results exactly. There could be three possible reasons for this. One is that the $\mathrm{RF}$ field inside the coil is not uniform enough, the second is that $\mathrm{YBa}_{2} \mathrm{Cu}_{3} \mathrm{O}_{7}$ is not perfectly oriented, and the third is that, while the average $\eta$ in $\mathrm{YBa}_{2} \mathrm{Cu}_{3} \mathrm{O}_{7}$ is zero, fluctuation are important due to charge inhomogeneity. Further study is required to address these possibilities.

\section{CONCLUDING REMARKS}

We have demonstrated that ADNQR could be used to determine $\eta$ and therefore $\nu_{q}$ at particular frequencies along a broad NQR line. In a future publication we will use ADNQR to determine the charge fluctuations evolution as a function of doping in high temperature superconductors.

\section{ACKNOWLEDGMENTS}

This project was funded by the Israeli Science Foundation.
In order to appreciate the influence of a non perfect pulses on the echo intensity, we examine the situation for a single spin $1 / 2$. In a two pulse experiment, in which the second pulse is twice as long as the first one, the nuclear spin expectation value at the time of the echo is given by

$$
\begin{aligned}
\left\langle I_{x}\right\rangle_{2 \tau} & =\operatorname{Tr}\left\{\frac{\exp (-\beta \mathcal{H})}{Z}\right. \\
& \exp \left(-i I_{x} \theta\right) \exp \left(-i \omega I_{z} \tau\right) \exp \left(-2 i I_{x} \theta\right) \exp \left(-i \omega I_{z} \tau\right) I_{x} \\
& \left.\exp \left(i \omega I_{z} \tau\right) \exp \left(2 i I_{x} \theta\right) \exp \left(i \omega I_{z} \tau\right) \exp \left(i I_{x} \theta\right)\right\}
\end{aligned}
$$

where $\theta=\omega_{1} t_{\pi / 2}$. In the high temperature approximation this simplifies to

$$
\left\langle I_{x}\right\rangle_{2 \tau}=\frac{\beta \omega}{4} \sin \theta\left[\sin ^{2} \theta-\cos ^{2} \theta\left\{2 \cos \left(\omega_{0} \tau\right)+\cos \left(\omega_{0} 2 \tau\right)\right\}\right]
$$

The oscillating term which depends on $\tau$ is a residue of the second pulse, and the one which depends on $2 \tau$ is a result of the first pulse. They contribute only if these pulses are not perfect, namely $\theta \neq \pi / 2$. In reality, these oscillating terms will relax before the echo is formed even if the pulses are not perfect. So if $\tau$ is long enough

$$
\left\langle I_{x}\right\rangle_{2 \tau}=\frac{\beta \omega}{4} \sin ^{3} \theta
$$

This is the origin of the cubic pulse length dependence of the signal as a function of the RF field.

[1] N. Sinyavsky, M. Ostafin, and M. Maćkowiak Z. Naturforsch 51a, 363 (1996). H. Robert and D. Pusiol, J. Chem. Phys. 106 (6), 2096 (1997).

[2] G. S. Harbison, A. Slokenbergs, and T. M. Barbara, J. Chem. Phys. 90 (10), 5292 (1989).

[3] F. V. Chávez, F. Casanova, H. Robert, and D. Pusiol, J. Chem. Phys. 108 (5) 181 (1998).

[4] J. C. Pratt, Molecular Physics, 1977, Vol. 34, No. 2, 539555.

[5] P. M. Singer, A. W. Hunt. and T. Imai, Phys. Rev. Lett 88, 047602 (2002).

[6] T. P. Das and E. L. Hahn, Solid State Physics, Suppl 1 (Academic Press), 1958.

[7] A. J. Vega et al., Phys. Rev. B 39, 2322 (1989).

[8] C. H. Pennington, D. J. Durand, D. B. Zax, and C. P. Slichter, Phys. Rev. B 37, 7944 (1988). H. Lutgemeier et al., Physica C 153-155, 731 (1988), Tadashi Shimizu et al., Journal of the Physical Society of Japan, 57, 2494 (1988). 